\title{
Shower and Slow Particle Productions in Nucleus-Nucleus Collisions at High Energy
}

\author{
S. Fakhraddin,, Magda A. Rahim, ${ }^{1,2}$ and Hamdy M. M. Zayd \\ ${ }^{1}$ Physics Department, Faculty of Science, Sana'a University, Sana'a, Yemen \\ ${ }^{2}$ Physics Department, Faculty of Science \& Arts (Girls Division), Najran University, Najran, Saudi Arabia \\ Correspondence should be addressed to S. Fakhraddin; sakinafal@hotmail.com
}

Received 11 December 2013; Accepted 17 February 2014; Published 7 April 2014

Academic Editor: Chen Wu

Copyright (C) 2014 S. Fakhraddin et al. This is an open access article distributed under the Creative Commons Attribution License, which permits unrestricted use, distribution, and reproduction in any medium, provided the original work is properly cited. The publication of this article was funded by SCOAP . $^{3}$

\begin{abstract}
The multiplicity distributions of shower, grey, and black particles produced in interactions of ${ }^{4} \mathrm{He},{ }^{12} \mathrm{C},{ }^{16} \mathrm{O},{ }^{22} \mathrm{Ne}$, and ${ }^{28} \mathrm{Si}$ with emulsion $(\mathrm{Em})$ at $4.1-4.5 \mathrm{~A} \mathrm{GeV} / \mathrm{c}$ beam energies, and their dependence on target groups $(\mathrm{H}, \mathrm{CNO}$, and $\mathrm{AgBr})$ is presented and has been reproduced by multisource thermal model. The multiplicity and the angular distributions of the three types of particles have been investigated. The experimental results are compared with the corresponding ones from the model. We found that the experimental data agrees with theoretical calculations using multisource thermal model.
\end{abstract}

\section{Introduction}

Study of the secondary charged particles produced in heavy ion collisions is attracting a great deal of attention during the recent ten years. Since the first run of the Dubna Synchrophasotron, in 1980, a lot of data for nuclear fragmentation in light- and heavy-ion collisions at high energy have been collected $[1-5]$. The measurements show that the average multiplicity of shower, grey particles increases with increase in projectile mass, but the average multiplicity of black particles is approximately constant. These observations have generated a flurry of theoretical activities [6-11].

Many models have been introduced to describe the multiparticle production in the nucleus-nucleus $(\mathrm{N}-\mathrm{N})$ interactions; some of them concern the dynamical evolution of interacting systems [12-18]. Others concern the thermal characteristics of final-state particles and fragments. One of these thermal models is the multisource thermal model, proposed to explain the multiplicity and angular distributions, based on the assumption that many emission sources are assumed to be formed in the interactions [19-30]. The aim of the present research is to check the model validity for describing the basic characteristics of particle production in the interactions of nuclei with emulsion at $4.1-4.5 \mathrm{~A} \mathrm{GeV} / \mathrm{c}$, mainly beams of
4.1 A GeV/c ${ }^{22} \mathrm{Ne}$ and $4.5 \mathrm{~A} \mathrm{GeV} / \mathrm{c}\left({ }^{4} \mathrm{He},{ }^{12} \mathrm{C},{ }^{16} \mathrm{O}\right.$, and $\left.{ }^{28} \mathrm{Si}\right)$ from Dubna Synchrophasotron.

\section{Multisource Thermal Model}

2.1. Multiplicity Distribution. The physics picture of the following discussions is based on the multisource thermal model [31-34], which is mainly used in the descriptions of particle (fragment) emission angles, azimuthal angles, and transverse flows in nucleus-nucleus (NN) collisions. In the model, many emission sources of particles and fragments are assumed to be formed in high energy collisions. According to the interaction mechanisms or event sample, the sources are divided into $l$ groups (subsamples). The source number in the $j$ th group is assumed to be $m_{j}$. Each source contributes multiplicity distribution to be an exponential distribution.

The multiplicity $\left(n_{i j}\right)$ distribution contributed by the $i$ th source in the $j$ th group is an exponential function:

$$
P_{i j}\left(n_{i j}\right)=\frac{1}{\left\langle n_{i j}\right\rangle} \exp \left(-\frac{n_{i j}}{\left\langle n_{i j}\right\rangle}\right),
$$

where

$$
\left\langle n_{i j}\right\rangle=\int n_{i j} p_{i j}\left(n_{i j}\right) d n_{i j}
$$


is the mean multiplicity contributed by the $i$ th source in the $j$ th group. As in [31], we assume that

$$
\left\langle n_{1 j}\right\rangle=\left\langle n_{2 j}\right\rangle=\left\langle n_{3 j}\right\rangle=\cdots\left\langle n_{m_{j} j}\right\rangle .
$$

The multiplicity $\left(n_{\mathrm{ch}}\right)$ distribution contributed by the $j$ th group is the fold of $m_{j}$ exponential functions; that is,

$$
P_{j}\left(n_{\mathrm{ch}}\right)=\frac{n_{\mathrm{ch}}^{m_{j}-1}}{\left(m_{j}-1\right) !\left\langle n_{i j}\right\rangle^{m_{j}}} \exp \left(-\frac{n_{\mathrm{ch}}}{\left\langle n_{i j}\right\rangle}\right) \text {. }
$$

It is an Erlang distribution. The total multiplicity distribution contributed by the $l$ groups can be written as

$$
P_{j}\left(n_{\mathrm{ch}}\right)=\frac{1}{N} \frac{d N}{d N_{\mathrm{ch}}}=\sum_{j=1}^{l} k_{j} P_{j}\left(n_{\mathrm{ch}}\right),
$$

where $k_{j}$ is the relative weight contributed by the $j$ th group.

In the Monte Carlo calculation, let $R_{i j}$ denote random variable in $[0,1]$. For the $j$ th group, we have

$$
\begin{gathered}
n_{i j}=-\left\langle n_{i j}\right\rangle \ln R_{i j}, \\
n_{\mathrm{ch}}=-\sum_{i=1}^{m_{j}}\left\langle n_{i j}\right\rangle \ln R_{i j} .
\end{gathered}
$$

The multiplicity distribution is obtained by a statistical method. Meanwhile, the mean multiplicities contributed by the $j$ th group and the $l$ groups are given by

$$
\begin{gathered}
\left\langle n_{\mathrm{ch}}\right\rangle=m_{j}\left\langle n_{i j}\right\rangle, \\
\left\langle n_{\mathrm{ch}}\right\rangle=\sum_{j=1}^{l} k_{j} m_{j}\left\langle n_{i j}\right\rangle,
\end{gathered}
$$

respectively.

Generally speaking [35], $j=1$ for $e^{-} e^{+}, p p\left(p p^{-}\right)$, and $e^{-} p$ collisions at not too high energies (less than a few hundred $\mathrm{GeV})$. The parameters for the collisions are $\left\langle n_{i j}\right\rangle$ and $m_{j}$. For the mentioned collisions at very high energies (greater than a few hundred $\mathrm{GeV}$ ), $j=2$ or 3 due to the different interaction mechanisms existing in the event samples. For $p A$ and $\mathrm{N}-\mathrm{N}$ collisions at a fixed impact parameter, $m_{j}$ can be regarded as the number of participant nucleons. The weight $k_{j}$ in (5) is obtained by the geometrical weight of the impact parameter. This formula was first proposed by Liu et al. to describe the multiplicity distributions of final-state particles produced in "elementary" particle interactions and heavy ion collisions at high energies. The basis of the formula is a multisource model and each source contributes multiplicity distribution to be an exponential form. The model treats uniformly the finalstate particles and nuclear fragments by the same formula. It is shown that the model is successful in the descriptions of multiplicity distributions of different types of particles and projectile fragments.

2.2. Emission Angle of Particles. According to the multisource thermal model suggested by Liu et al. [31-36], many emission sources are assumed to be formed in the interactions. Let the beam direction of the incoming projectile be the $o z$-axis, and let the reaction plane be the $x o z$ plane. Each source is assumed to emit particles isotropically in the source rest frame. As the first approximation, the three components $p_{x, y, z}^{\backslash}$ of the particle momentum in the source rest frame are assumed to obey Gaussian distributions with the same deviation width $\sigma$ [37]. Considering the motion of the emission source and the interactions among emission sources, the particle momentum components $p_{x}, p_{y}$, and $p_{z}$ in the final state in the laboratory reference frame are different from those in the rest frame of the emission source. The simplest relations between $p_{x}$ and $p_{x}^{\backslash}, p_{y}$ and $p_{y}^{\backslash}$, and $p_{z}$ and $p_{z}^{\backslash}$ are linear:

$$
\begin{aligned}
& p_{x}=a_{x} p_{x}^{\backslash}+B_{x}=a_{x} p_{x}^{\backslash}+a_{x} \sigma_{i} \\
& p_{y}=a_{y} p_{y}^{\backslash}+B_{y}=a_{y} p_{y}^{\backslash}+a_{y} \sigma_{i} \\
& p_{z}=a_{z} p_{z}^{\backslash}+B_{z}=a_{z} p_{z}^{\backslash}+a_{z} \sigma_{i}
\end{aligned}
$$

where $B_{x}, B_{y}$, and $B_{z}$ are free parameters and $\sigma_{i}$ is the parameter that characterizes the width of the momentum distribution in the source reference frame. $a_{x}, b_{x}, a_{y}, b_{y}, a_{z}$, and $b_{z}$ are free parameters. Let $R_{1}, R_{2}, R_{3}, R_{4}, R_{5}$, and $R_{6}$ denote random variables distributed in $[0,1]$; we have

$$
\begin{aligned}
& p_{x}^{\backslash}=\sqrt{-2 \ln R_{1}} \cos \left(2 \pi R_{2}\right) \sigma_{i}, \\
& p_{y}^{\prime}=\sqrt{-2 \ln R_{3}} \cos \left(2 \pi R_{4}\right) \sigma_{i}, \\
& p_{z}^{\prime}=\sqrt{-2 \ln R_{5}} \cos \left(2 \pi R_{6}\right) \sigma_{i}
\end{aligned}
$$

because $p_{x}^{\backslash}, p_{y}^{\backslash}$, and $p_{z}^{\backslash}$ obey a Gaussian distribution law.

The emission angle $\theta$ of a target fragment in the laboratory reference frame is given by

$$
\theta=\arctan \frac{\sqrt{p_{x}^{2}+p_{y}^{2}}}{p_{z}}
$$

Considering (9)-(11), we have

$$
\theta=\arctan \frac{\sqrt{\left[a_{x} \sqrt{-2 \ln R_{1}} \cos \left(2 \pi R_{2}\right)+b_{x}\right]^{2}+\left[a_{y} \sqrt{-2 \ln R_{3}} \cos \left(2 \pi R_{4}\right)+b_{y}\right]^{2}}}{a_{z} \sqrt{-2 \ln R_{5}} \cos \left(2 \pi R_{6}\right)+b_{z}} .
$$


TABLE 1: The average multiplicities of the charged secondary particles emitted in ${ }^{4} \mathrm{He}-,{ }^{12} \mathrm{C}-,{ }^{16} \mathrm{O}-,{ }^{22} \mathrm{Ne}-$, and ${ }^{28} \mathrm{Si}$-Em interactions and those emitted in the interactions with the different groups of target nuclei $(\mathrm{H}, \mathrm{CNO}, \mathrm{Em}$, and $\mathrm{AgBr})$ compared with those obtained by the model.

\begin{tabular}{|c|c|c|c|c|c|c|}
\hline Projectile & & $\left\langle n_{i}\right\rangle$ & $\mathrm{H}$ & $\mathrm{CNO}$ & $\mathrm{Em}$ & $\mathrm{AgBr}$ \\
\hline \multirow{6}{*}{${ }^{4} \mathrm{He}$} & MSTM & $\left\langle n_{s}\right\rangle$ & 1.623 & 3.3263 & 3.903 & 5.78 \\
\hline & EXP & $\left\langle n_{s}\right\rangle$ & $1.619 \pm 0.1$ & $3.112 \pm 0.133$ & $3.757 \pm 0.096$ & $5.77 \pm 0.151$ \\
\hline & MSTM & $\left\langle n_{g}\right\rangle$ & 0.288 & 2.35 & 5.372 & 9.474 \\
\hline & EXP & $\left\langle n_{g}\right\rangle$ & $0.288 \pm 0.03$ & $2 \pm 0.066$ & $4.3899 \pm 0.17$ & $9.38 \pm 0.3$ \\
\hline & MSTM & $\left\langle n_{b}\right\rangle$ & 0.163 & 2.5 & 4.86 & 9.519 \\
\hline & EXP & $\left\langle n_{b}\right\rangle$ & $0.172 \pm 0.024$ & $2.25 \pm 0.07$ & $4.395 \pm 0.149$ & $9.223 \pm 0.21$ \\
\hline \multirow{6}{*}{${ }^{12} \mathrm{C}$} & MSTM & $\left\langle n_{s}\right\rangle$ & 2.014 & 6.22 & 8.897 & 13.975 \\
\hline & EXP & $\left\langle n_{s}\right\rangle$ & $2.062 \pm 0.186$ & $6 \pm 0.25$ & $8.0 \pm 0.24$ & $13.975 \pm 0.4$ \\
\hline & MSTM & $\left\langle n_{g}\right\rangle$ & 0.288 & 2.35 & 5.274 & 10.882 \\
\hline & EXP & $\left\langle n_{g}\right\rangle$ & $0.293 \pm 0.03$ & $2.04 \pm 0.1$ & $5.081 \pm 0.22$ & $10.753 \pm 0.4$ \\
\hline & MSTM & $\left\langle n_{b}\right\rangle$ & 0.205 & 2.5 & 4.6346 & 9.519 \\
\hline & EXP & $\left\langle n_{b}\right\rangle$ & $0.204 \pm 0.027$ & $2.28 \pm 0.075$ & $4.57 \pm 0.16$ & $9.293 \pm 0.21$ \\
\hline \multirow{6}{*}{${ }^{16} \mathrm{O}$} & MSTM & $\left\langle n_{s}\right\rangle$ & 2.196 & 6.621 & 9.741 & 16.664 \\
\hline & EXP & $\left\langle n_{s}\right\rangle$ & $2.454 \pm 0.145$ & $6.679 \pm 0.197$ & $9.417 \pm 0.206$ & $16.426 \pm 0.349$ \\
\hline & MSTM & $\left\langle n_{g}\right\rangle$ & 0.268 & 2.35 & 6.284 & 12.6 \\
\hline & EXP & $\left\langle n_{g}\right\rangle$ & $0.264 \pm 0.02$ & $2.154 \pm 0.06$ & $6.353 \pm 0.17$ & $12.454 \pm 0.29$ \\
\hline & MSTM & $\left\langle n_{b}\right\rangle$ & 0.243 & 2.5 & 4.315434 & 9.217 \\
\hline & EXP & $\left\langle n_{b}\right\rangle$ & $0.24 \pm 0.02$ & $2.17 \pm 0.05$ & $4.421 \pm 0.097$ & $8.58 \pm 0.13$ \\
\hline \multirow{6}{*}{${ }^{22} \mathrm{Ne}$} & MSTM & $\left\langle n_{s}\right\rangle$ & 2.11 & 6.605 & 10.769 & 18.919 \\
\hline & EXP & $\left\langle n_{s}\right\rangle$ & $2.24 \pm 0.0898$ & $6.66 \pm 0.151$ & $10.254 \pm 0.113$ & $18.482 \pm 0.29$ \\
\hline & MSTM & $\left\langle n_{g}\right\rangle$ & 0.249 & 2.35 & 6.752 & 13.547 \\
\hline & EXP & $\left\langle n_{g}\right\rangle$ & $0.252 \pm 0.013$ & $2.2 \pm 0.04$ & $6.188 \pm 0.295$ & $13.495 \pm 0.214$ \\
\hline & MSTM & $\left\langle n_{b}\right\rangle$ & 0.243 & 2.301 & 4.288 & 8.978 \\
\hline & EXP & $\left\langle n_{b}\right\rangle$ & $0.238 \pm 0.013$ & $2.05 \pm 0.034$ & $4.151 \pm 0.103$ & $8.482 \pm 0.09$ \\
\hline \multirow{6}{*}{${ }^{28} \mathrm{Si}$} & MSTM & $\left\langle n_{s}\right\rangle$ & 2.461 & 7.836 & 11.776 & 21.007 \\
\hline & EXP & $\left\langle n_{s}\right\rangle$ & $2.887 \pm 0.22$ & $7.51 \pm 0.297$ & $11.784 \pm 0.343$ & $20.163 \pm 0.602$ \\
\hline & MSTM & $\left\langle n_{g}\right\rangle$ & 0.249 & 2.39 & 6.071 & 13.156 \\
\hline & EXP & $\left\langle n_{g}\right\rangle$ & $0.2474 \pm 0.03$ & $2.266 \pm 0.07$ & $6.405 \pm 0.228$ & $13.224 \pm 0.383$ \\
\hline & MSTM & $\left\langle n_{b}\right\rangle$ & 0.163 & 2.5 & 4.678 & 9.504 \\
\hline & EXP & $\left\langle n_{b}\right\rangle$ & $0.165 \pm 0.022$ & $2.2 \pm 0.064$ & $4.449 \pm 0.1244$ & $8.651 \pm 0.17$ \\
\hline
\end{tabular}

\section{Results and Discussions}

3.1. Multiplicity Characteristics. To study the multiplicity behavior of the target fragmentation as function of mass number of the target nucleus $A_{T}$, we classify the emulsion nuclei based on $n_{h}\left(n_{h}=n_{g}+n_{b}\right)$ into three groups [38]: two types of light nuclei ( $\mathrm{H}$ and $\mathrm{CNO}$ ) and one type of heavy nuclei (AgBr). Collisions with $\mathrm{H}$ target nuclei are events with $n_{h}=0,1$, collisions with only one bound nucleon in CNO or $\mathrm{AgBr}$ target nuclei these events having $2 \leq n_{h} \leq 7$ are mostly interactions with CNO targets with some admixture of peripheral $\mathrm{AgBr}$ interactions. All events with $n_{h} \geq 8$ are only due to $\mathrm{AgBr}$ interactions. It should be noted that the classification of events in emulsion is not unique; however, there is no perfect method for classifying events due to the limitations of the emulsion technique [39].

Table 1 illustrates the average values of shower, grey, black, and heavy particles produced in interactions of different projectiles with Em at momentum 4.1-4.5 A GeV/c. The experimental data has been taken from [40] and the data available from the High Energy Physics Group at Sana'a University.

The comparison between the average values of the multiplicities $\left\langle n_{s}\right\rangle,\left\langle n_{g}\right\rangle$, and $\left\langle n_{b}\right\rangle$ obtained experimentally and those obtained by multisource thermal model shows a fair agreement between the model and the experiment for a wide range of projectiles.

It can be noticed from this table that the values of $\left\langle n_{b}\right\rangle$ are nearly independent of the nature of incident projectiles, while $\left\langle n_{s}\right\rangle,\left\langle n_{g}\right\rangle$, and $\left\langle n_{h}\right\rangle$ show their dependence on the projectile mass number $A_{p}$. This fact indicates that the target evaporation fragments do not seem to depend on the mass of the projectile.

Figure 1 presents the multiplicity distributions of shower particles in interactions of ${ }^{28} \mathrm{Si},{ }^{22} \mathrm{Ne},{ }^{16} \mathrm{O},{ }^{12} \mathrm{C}$, and ${ }^{4} \mathrm{He}$ with (a) Em, (b) H, (c) CNO, and (d) AgBr. For comparison, 


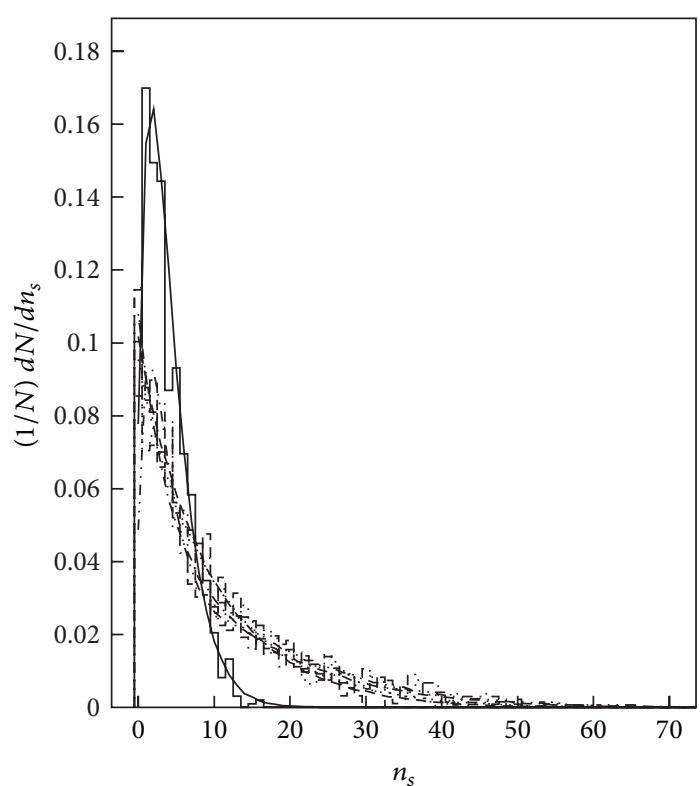

(a)

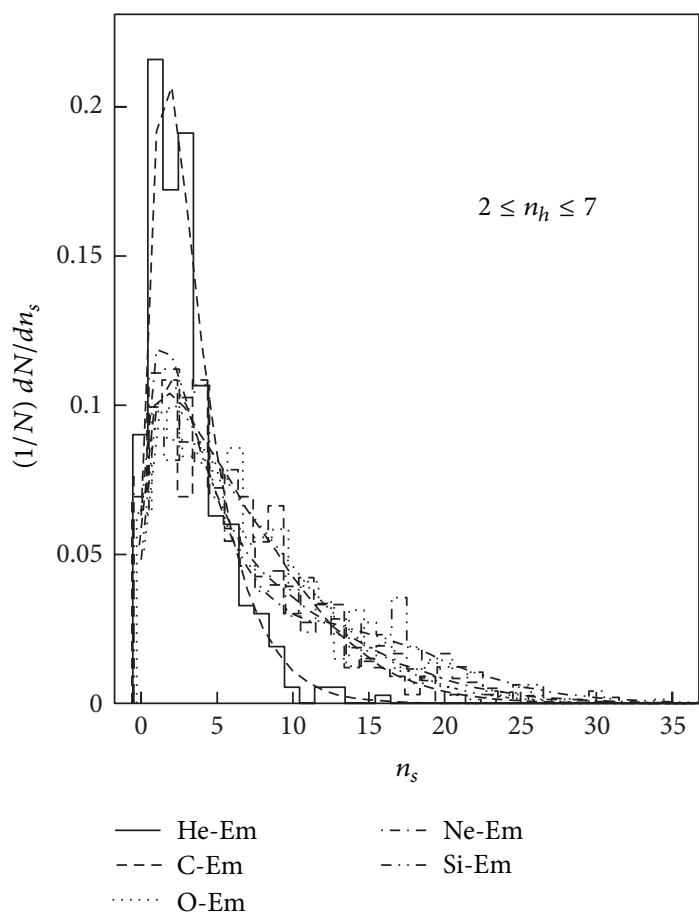

(c)

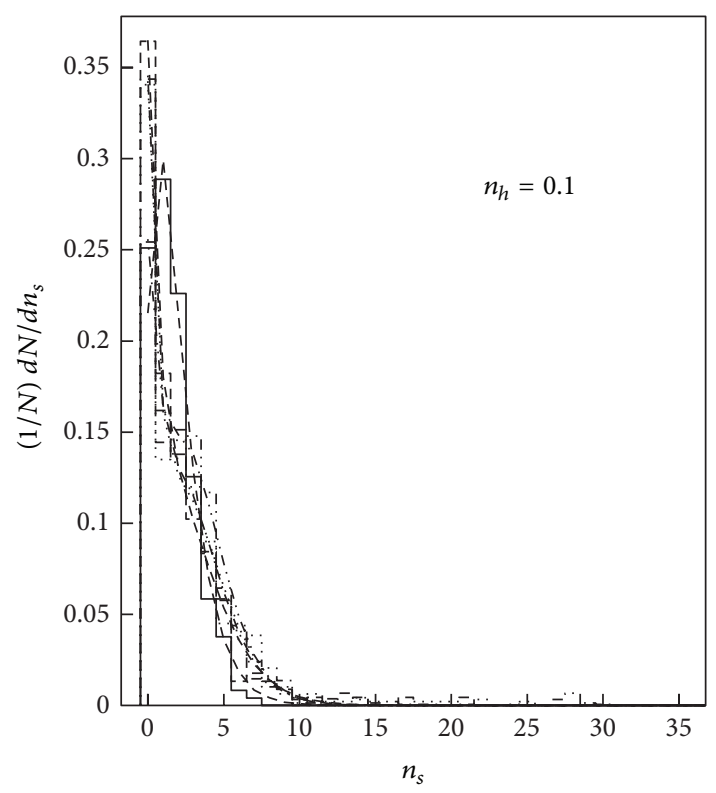

(b)

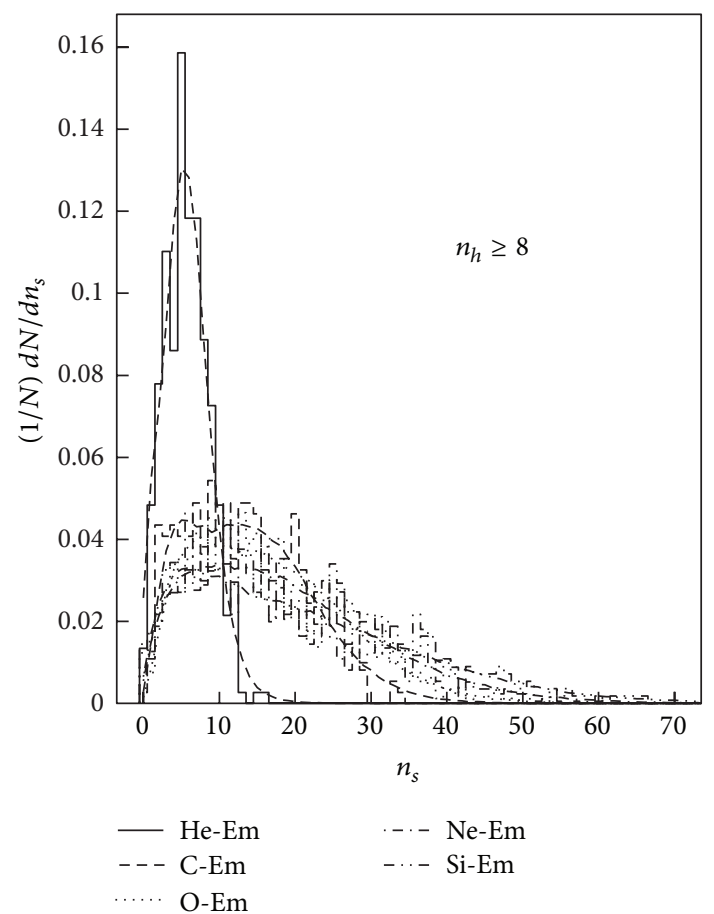

(d)

Figure 1: The normalized multiplicity distributions for shower particles produced in the interactions of projectiles with (a) Em, (b) H, (c) $\mathrm{CNO}$, and (d) $\mathrm{AgBr}$, respectively. The histograms represent the experimental data, while the curves are the corresponding ones calculated according to the multisource thermal model.

distributions obtained by the multisource thermal model calculations are also shown as curves. All the distributions are normalized to one. ${ }^{4} \mathrm{He}$ is represented by solid histogram, ${ }^{12} \mathrm{C}$ by dashed one, ${ }^{16} \mathrm{O}$ by dotted one, ${ }^{22} \mathrm{Ne}$ by dash-dotted one, and ${ }^{28} \mathrm{Si}$ by beaded one. It can be noticed from this figure that the model is in good agreement with the experimental data for the projectiles. The height of the multiplicity distribution of $n_{s}$ decreases with increase in projectile mass, while the position of the peak moves to higher multiplicities with increasing $A_{T}$. The distributions get wider with increasing $A_{T}$ 


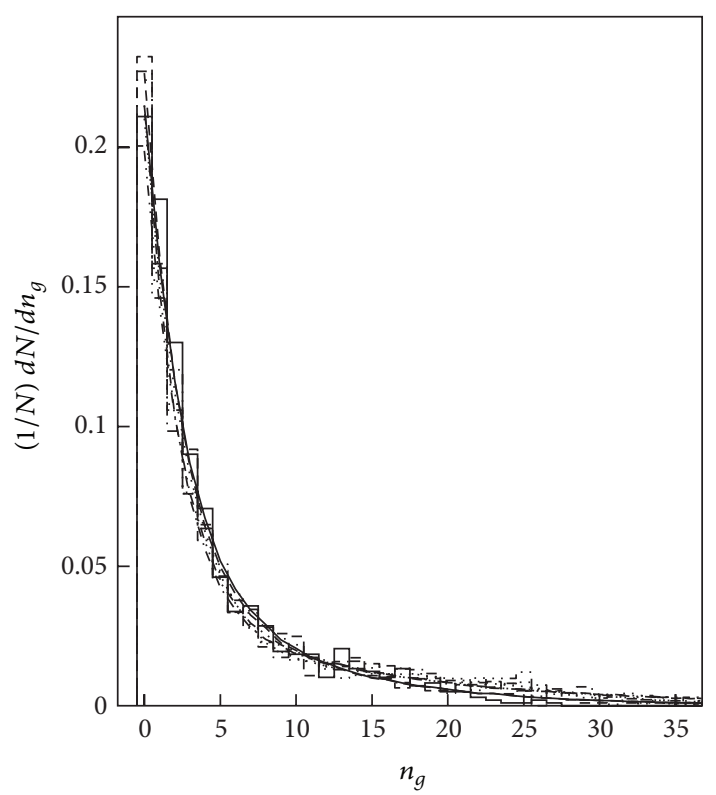

(a)

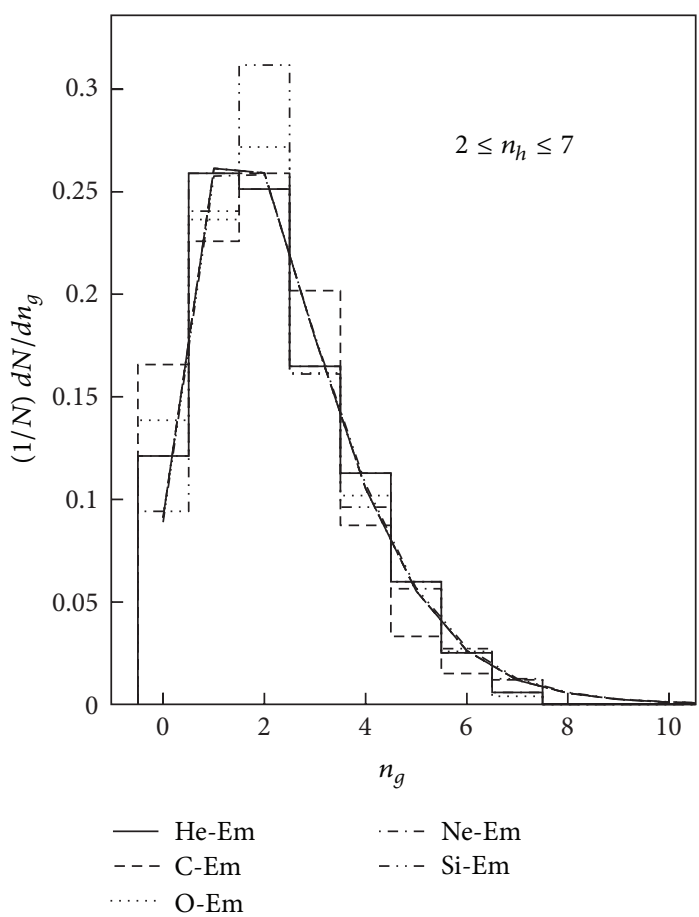

(c)

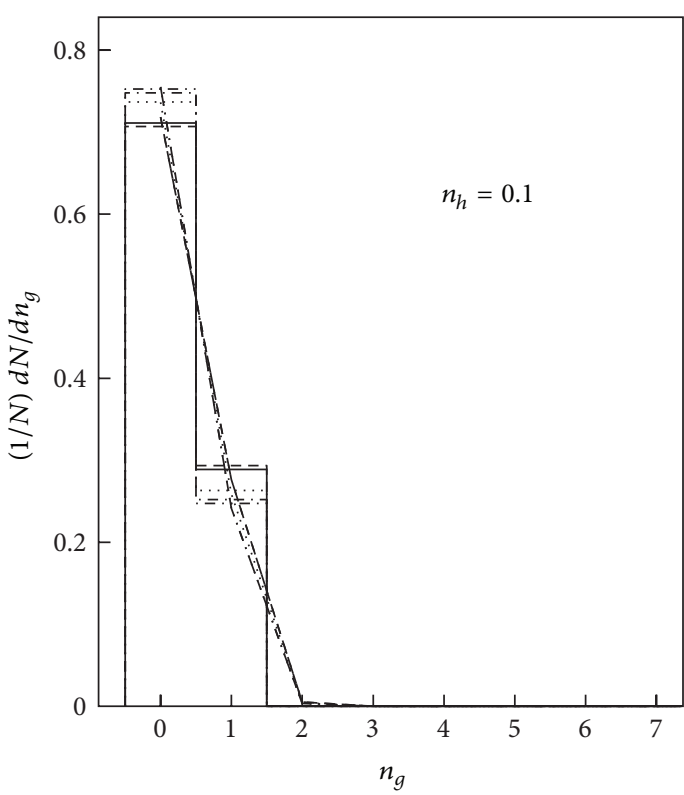

(b)

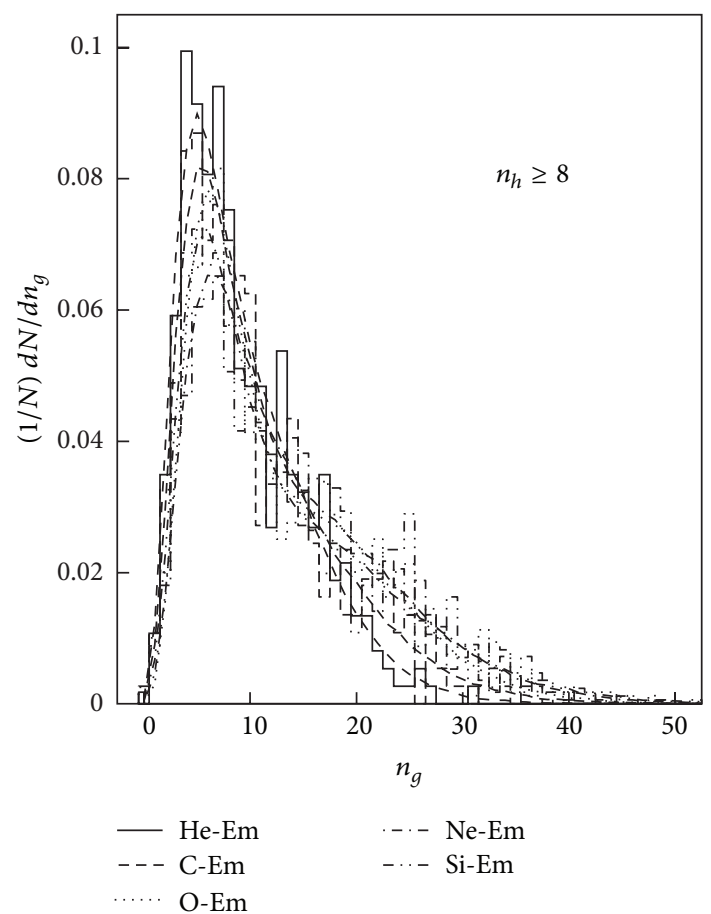

(d)

Figure 2: The normalized multiplicity distributions for the grey particles produced in the interactions of projectiles with (a) Em, (b) H, (c) $\mathrm{CNO}$, and (d) $\mathrm{AgBr}$, respectively. The histograms represent the experimental data, while the curves are the corresponding ones calculated according to the multisource thermal model.

where the distributions have larger tails. This may reflect the effect of the target mass number on the number of collisions of ${ }^{28} \mathrm{Si},{ }^{22} \mathrm{Ne},{ }^{16} \mathrm{O},{ }^{12} \mathrm{C}$, and ${ }^{4} \mathrm{He}$ with the target nuclei.

Similarly the multiplicity distributions of grey particles are illustrated in Figure 2. It can be seen that the dependence of the height of the distributions on $A_{p}$ gets weaker than that in case of shower particles. Also one can notice that the distributions for ${ }^{28} \mathrm{Si},{ }^{22} \mathrm{Ne},{ }^{16} \mathrm{O},{ }^{12} \mathrm{C}$, and ${ }^{4} \mathrm{He}$ with $\mathrm{AgBr}$ interactions are broader than those for ${ }^{28} \mathrm{Si},{ }^{22} \mathrm{Ne},{ }^{16} \mathrm{O},{ }^{12} \mathrm{C}$, and ${ }^{4} \mathrm{He}$ with Em, $\mathrm{H}$, and CNO.

The same features can be observed for black particles $\left(n_{b}\right)$ distributions given in Figure 3 as for $n_{g}$. Figure 3(a) shows 


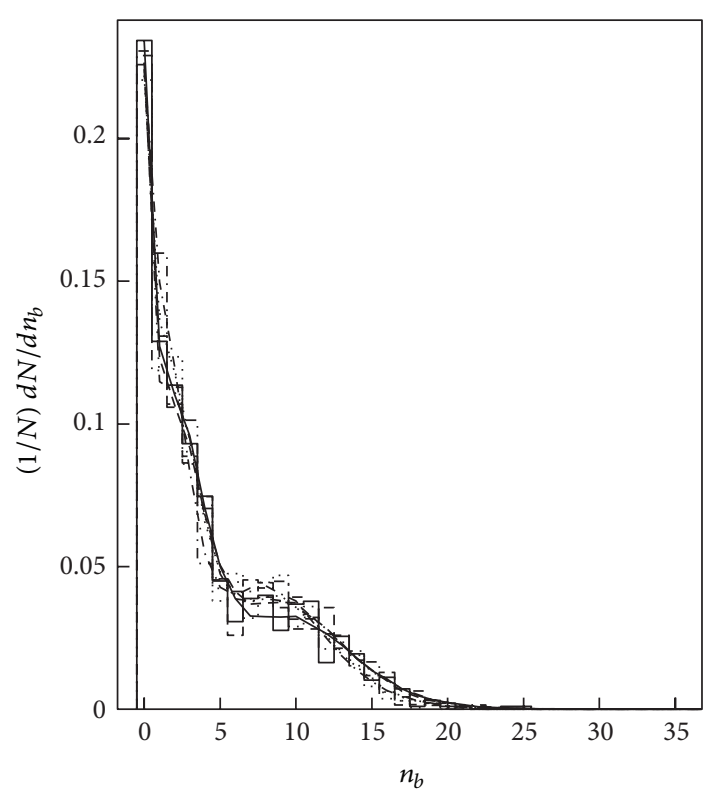

(a)

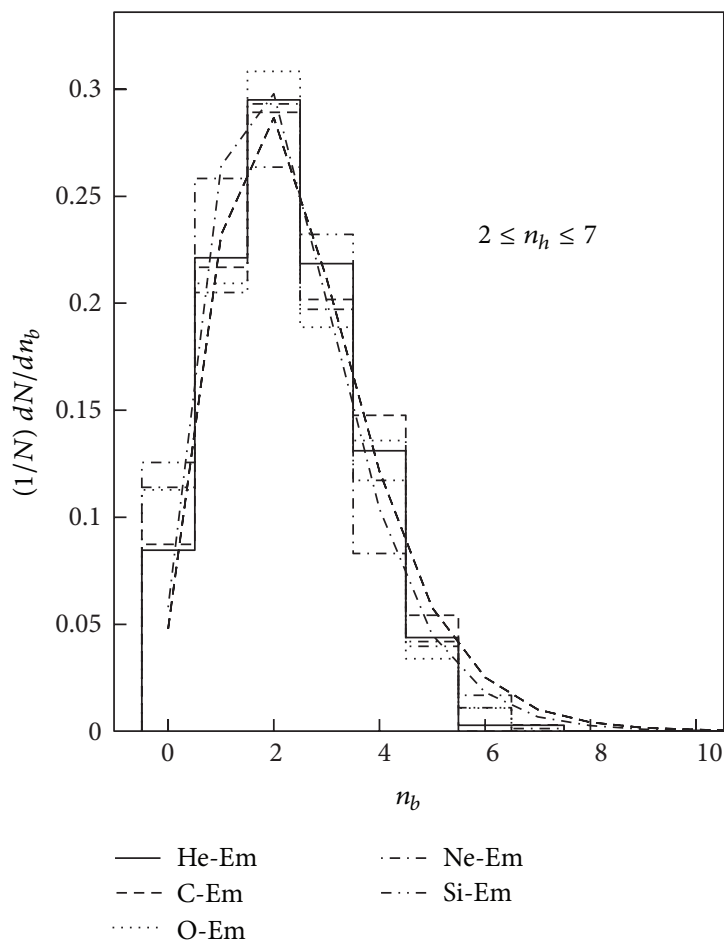

(c)

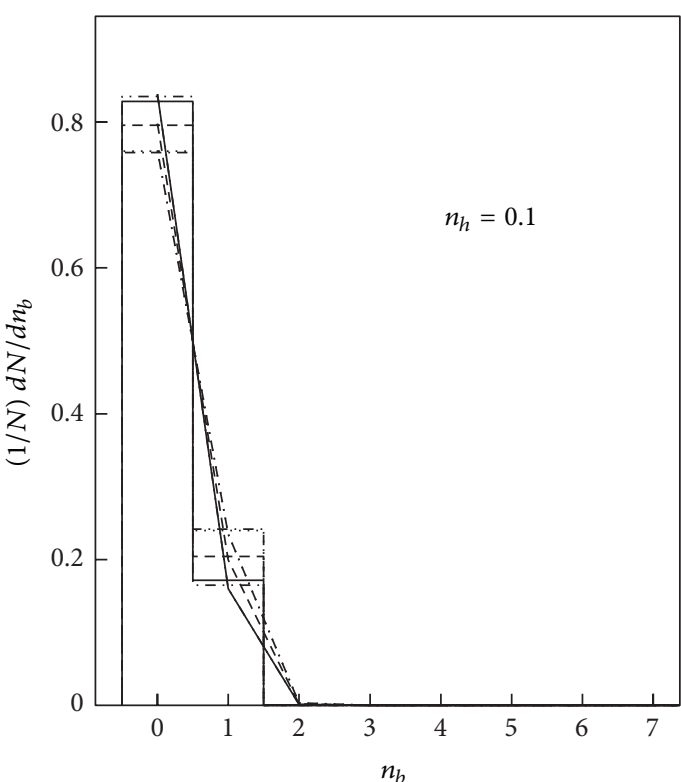

(b)

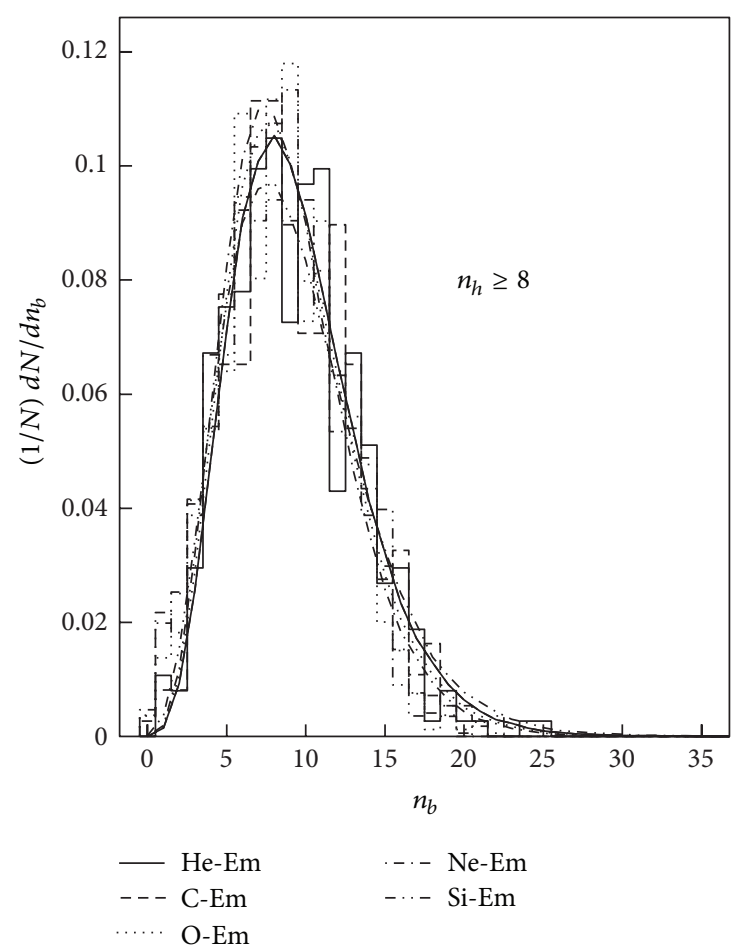

(d)

FIGURE 3: The normalized multiplicity distributions for black particles produced in the interactions of projectiles with (a) Em, (b) H, (c) CNO, and (d) AgBr, respectively. The histograms represent the experimental data, while the curves are the corresponding ones calculated according to the multisource thermal model.

a tow-peak structure. The two peaks are around 0 and 10; this could be due to interactions with light- and heavy-target nuclei, respectively.

Finally, in Figure 4, we investigate the heavy particles $\left(n_{h}=n_{g}+n_{b}\right)$ distribution. Two obvious peaks are observed here, one around multiplicity 0 and the other one at 5 . The first peak could be due to interactions with $\mathrm{H}$ and the second one with CNO. There is one around 20, but it is flattened, and this could represent the collision with $\mathrm{AgBr}$. In all the above distributions, the model can reproduce 


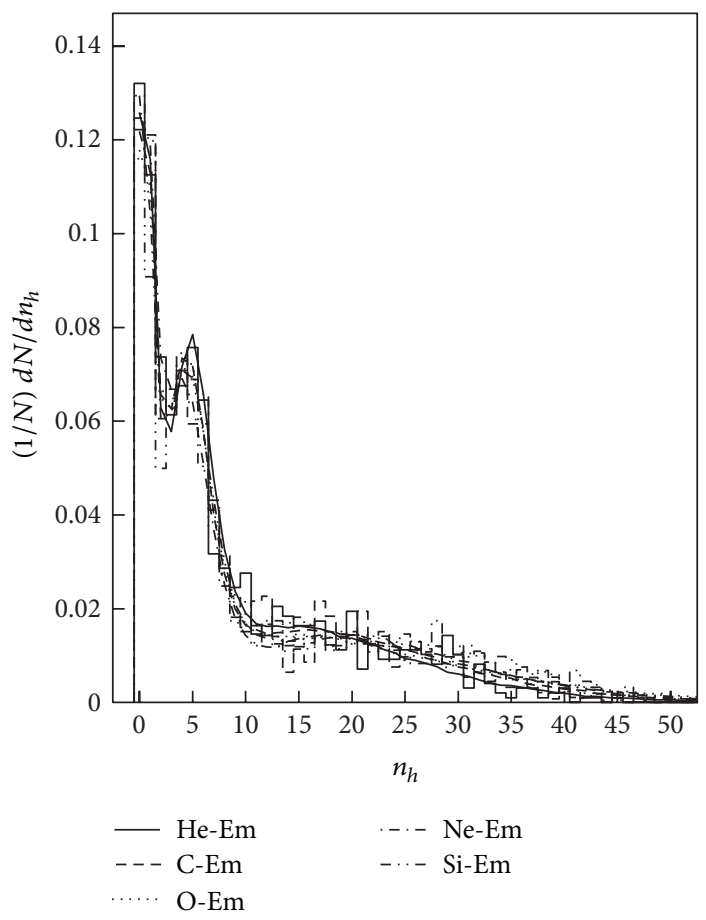

FIGURE 4: Normalized multiplicity distributions for heavy particles produced in the interactions of projectiles with Em at (4.1-4.5) A GeV/c. The histograms represent the experimental data, while the curves are the corresponding ones calculated according to the multisource thermal model.

TABLE 2: Parameter values for multiplicity distributions for shower, grey, black, and heavy particles. The values of $\left\langle n_{i 1}\right\rangle,\left\langle n_{i 2}\right\rangle$, and $\left\langle n_{i 3}\right\rangle$ and $m_{1}, m_{2}$, and $m_{3}$, and $k_{1}, k_{2}$, and $k_{3}$ for ${ }^{4} \mathrm{He}-,{ }^{12} \mathrm{C}-,{ }^{16} \mathrm{O}$-, and ${ }^{28} \mathrm{Si}-\mathrm{Em}$ at $4.5 \mathrm{~A} \mathrm{GeV} / \mathrm{c}$ and ${ }^{22} \mathrm{Ne}-\mathrm{Em}$ at $4.1 \mathrm{~A} \mathrm{GeV} / \mathrm{c}$.

\begin{tabular}{|c|c|c|c|c|c|c|c|c|c|c|c|}
\hline Projectile & & $\left\langle n_{i 1}\right\rangle$ & $\left\langle n_{i 2}\right\rangle$ & $\left\langle n_{i 3}\right\rangle$ & $m_{1}$ & $m_{2}$ & $m_{3}$ & $k_{1}$ & $k_{2}$ & $k_{3}$ & $\chi^{2} /$ dof \\
\hline \multirow{4}{*}{${ }^{4} \mathrm{He}$} & $n_{s}$ & 2.2 & - & - & 2 & - & - & 1 & 0 & 0 & 1.026 \\
\hline & $n_{g}$ & 2.5 & 9.2 & - & 1 & 1 & - & 0.5 & 0.5 & 0 & 1.379 \\
\hline & $n_{b}$ & 0.87 & 0.96 & 1.16 & 1 & 4 & 10 & 0.33 & 0.4 & 0.27 & 1.711 \\
\hline & $n_{h}$ & 0.74 & 0.59 & 5 & 2 & 10 & 4 & 0.32 & 0.32 & 0.36 & 1.663 \\
\hline \multirow{4}{*}{${ }^{12} \mathrm{C}$} & $n_{s}$ & 4.6 & 7.1 & - & 1 & 2 & - & 0.5 & 0.5 & 0 & 1.143 \\
\hline & $n_{g}$ & 2.3 & 9.2 & - & 1 & 1 & - & 0.5 & 0.5 & 0 & 1.232 \\
\hline & $n_{b}$ & 0.89 & 0.96 & 1.12 & 1 & 4 & 10 & 0.32 & 0.38 & 0.30 & 0.658 \\
\hline & $n_{h}$ & 0.75 & 0.63 & 5.5 & 2 & 9 & 4 & 0.31 & 0.38 & 0.31 & 1.416 \\
\hline \multirow{4}{*}{${ }^{16} \mathrm{O}$} & $n_{s}$ & 4.9 & 7.8 & - & 1 & 2 & - & 0.5 & 0.5 & 0 & 0.642 \\
\hline & $n_{g}$ & 2.45 & 5.38 & 3.1 & 1 & 1 & 7 & 0.42 & 0.42 & 0.26 & 0.887 \\
\hline & $n_{b}$ & 0.93 & 0.88 & 1.05 & 1 & 4 & 10 & 0.32 & 0.38 & 0.30 & 0.692 \\
\hline & $n_{h}$ & 0.74 & 0.63 & 7.75 & 2 & 9 & 3 & 0.31 & 0.31 & 0.38 & 0.611 \\
\hline \multirow{4}{*}{${ }^{22} \mathrm{Ne}$} & $n_{s}$ & 4.2 & 9.2 & - & 1 & 2 & - & 0.5 & 0.5 & 0 & 0.476 \\
\hline & $n_{g}$ & 2.2 & 14.2 & - & 1 & 1 & - & 0.5 & 0.5 & 0 & 0.688 \\
\hline & $n_{b}$ & 0.93 & 0.75 & 1.24 & 1 & 4 & 8 & 0.32 & 0.33 & 0.35 & 0.584 \\
\hline & $n_{h}$ & 0.74 & 0.81 & 4.83 & 2 & 7 & 5 & 0.32 & 0.32 & 0.36 & 0.43 \\
\hline \multirow{4}{*}{${ }^{28} \mathrm{Si}$} & $n_{s}$ & 1.8 & 9.3 & - & 2 & 2 & - & 0.42 & 0.58 & 0 & 0.917 \\
\hline & $n_{g}$ & 2.6 & 11 & - & 1 & 1 & - & 0.5 & 0.5 & 0 & 1.161 \\
\hline & $n_{b}$ & 0.8 & 0.7 & 1.35 & 1 & 5 & 8 & 0.31 & 0.35 & 0.34 & 1.84 \\
\hline & $n_{h}$ & 0.64 & 0.82 & 6 & 2 & 7 & 4 & 0.28 & 0.35 & 0.37 & 0.843 \\
\hline
\end{tabular}


TABLE 3: Parameter values for multiplicity distributions for shower, grey, and black particles. The values of $\left\langle n_{i 1}\right\rangle,\left\langle n_{i 2}\right\rangle, m_{1}, m_{2}$, and $k_{1}, k_{2}$ for ${ }^{4} \mathrm{He}-,{ }^{12} \mathrm{C}-,{ }^{16} \mathrm{O}-$, and ${ }^{28} \mathrm{Si}-\mathrm{H}$ at $4.5 \mathrm{~A} \mathrm{GeV} / \mathrm{c}$ and ${ }^{22} \mathrm{Ne}-\mathrm{H}$ at $4.1 \mathrm{~A} \mathrm{GeV} / \mathrm{c}$.

\begin{tabular}{|c|c|c|c|c|c|c|c|c|}
\hline Projectile & & $\left\langle n_{i 1}\right\rangle$ & $\left\langle n_{i 2}\right\rangle$ & $m_{1}$ & $m_{2}$ & $k_{1}$ & $k_{2}$ & $\chi^{2} /$ dof \\
\hline \multirow{3}{*}{${ }^{4} \mathrm{He}$} & $n_{s}$ & 1.16 & - & 2 & - & 1 & 0 & 1.248 \\
\hline & $n_{g}$ & 0.14 & - & 6 & - & 1 & 0 & 0.02 \\
\hline & $n_{b}$ & 0.12 & - & 6 & - & 1 & 0 & 0.049 \\
\hline \multirow{3}{*}{${ }^{12} \mathrm{C}$} & $n_{s}$ & 0.85 & 1.36 & 1 & 3 & 0.5 & 0.5 & 1.153 \\
\hline & $n_{g}$ & 0.14 & - & 6 & - & 1 & 0 & 0.039 \\
\hline & $n_{b}$ & 0.127 & - & 6 & - & 1 & 0 & 0.006 \\
\hline \multirow{3}{*}{${ }^{16} \mathrm{O}$} & $n_{s}$ & 0.9 & 1.1 & 1 & 4 & 0.5 & 0.5 & 1.622 \\
\hline & $n_{g}$ & 0.137 & - & 6 & - & 1 & 0 & 0.003 \\
\hline & $n_{b}$ & 0.133 & - & 6 & - & 1 & 0 & 0.004 \\
\hline \multirow{3}{*}{${ }^{22} \mathrm{Ne}$} & $n_{s}$ & 0.89 & 0.06 & 1 & 4 & 0.5 & 0.5 & 1.074 \\
\hline & $n_{g}$ & 0.134 & - & 6 & - & 1 & 0 & 0.02 \\
\hline & $n_{b}$ & 0.133 & - & 6 & - & 1 & 0 & 0.008 \\
\hline \multirow{3}{*}{${ }^{28} \mathrm{Si}$} & $n_{s}$ & 1.41 & 0.89 & 1 & 5 & 0.5 & 0.5 & 0.912 \\
\hline & $n_{g}$ & 0.134 & - & 6 & - & 1 & 0 & 0.006 \\
\hline & $n_{b}$ & 0.12 & - & 6 & - & 1 & 0 & 0.009 \\
\hline
\end{tabular}

TABLE 4: Parameter values for multiplicity distributions for shower, grey, and black particles. The values of $\left\langle n_{i 1}\right\rangle,\left\langle n_{i 2}\right\rangle, m_{1}, m_{2}$, and $k_{1}, k_{2}$ for ${ }^{4} \mathrm{He}-,{ }^{12} \mathrm{C}-,{ }^{16} \mathrm{O}$-, and ${ }^{28} \mathrm{Si}-\mathrm{CNO}$ at $4.5 \mathrm{~A} \mathrm{GeV} / \mathrm{c}$ and ${ }^{22} \mathrm{Ne}-\mathrm{CNO}$ at $4.1 \mathrm{~A} \mathrm{GeV} / \mathrm{c}$.

\begin{tabular}{|c|c|c|c|c|c|c|c|c|}
\hline Projectile & & $\left\langle n_{i 1}\right\rangle$ & $\left\langle n_{i 2}\right\rangle$ & $m_{1}$ & $m_{2}$ & $k 1$ & $k_{2}$ & $\chi^{2} /$ dof \\
\hline \multirow{3}{*}{${ }^{4} \mathrm{He}$} & $n_{s}$ & 0.9 & 1.62 & 3 & 3 & 0.5 & 0.5 & 1.783 \\
\hline & $n_{g}$ & 0.95 & - & 3 & - & 1 & 0 & 1.29 \\
\hline & $n_{b}$ & 0.75 & - & 4 & - & 1 & 0 & 1.41 \\
\hline \multirow{3}{*}{${ }^{12} \mathrm{C}$} & $n_{s}$ & 1.72 & 2.35 & 2 & 4 & 0.45 & 0.55 & 1.293 \\
\hline & $n_{g}$ & 0.95 & - & 3 & - & 1 & 0 & 1.027 \\
\hline & $n_{b}$ & 0.75 & - & 4 & - & 1 & 0 & 1.408 \\
\hline \multirow{3}{*}{${ }^{16} \mathrm{O}$} & $n_{s}$ & 1.75 & 2.52 & 2 & 4 & 0.45 & 0.55 & 1.223 \\
\hline & $n_{g}$ & 0.95 & - & 3 & - & 1 & 0 & 1.475 \\
\hline & $n_{b}$ & 0.75 & - & 4 & - & 1 & 0 & 1.853 \\
\hline \multirow{3}{*}{${ }^{22} \mathrm{Ne}$} & $n_{s}$ & 1.78 & 3 & 2 & 4 & 0.58 & 0.42 & 0.736 \\
\hline & $n_{g}$ & 0.95 & - & 3 & - & 1 & 0 & 1.29 \\
\hline & $n_{b}$ & 0.75 & - & 4 & - & 1 & 0 & 1.776 \\
\hline \multirow{3}{*}{${ }^{28} \mathrm{Si}$} & $n_{s}$ & 2.35 & 2.1 & 2 & 8 & 0.70 & 0.30 & 1.831 \\
\hline & $n_{g}$ & 0.96 & - & 3 & - & 1 & 0 & 0.136 \\
\hline & $n_{b}$ & 0.75 & - & 4 & - & 1 & 0 & 1.964 \\
\hline
\end{tabular}

the multiplicity characteristics for the different particles, and the experimental distributions are in agreement (within errors) with the theoretical ones.

The parameter values of $\left\langle n_{i 1}\right\rangle,\left\langle n_{i 2}\right\rangle$, and $\left\langle n_{i 3}\right\rangle, m_{1}, m_{2}$, and $m_{3}$, and $k_{1}, k_{2}$, and $k_{3}$ for multiplicity distributions for shower, grey, black, and heavy particles along with $\chi^{2} /$ dof are illustrated in Tables $2-5$. Table 2 shows these parameter values for Em, while the parameter values for $\mathrm{H}, \mathrm{CNO}$, and $\mathrm{AgBr}$ are given in Tables 3, 4, and 5, respectively.

3.2. Angular Distributions. The angular distributions for the different secondary charged particles $\theta_{s}, \theta_{g}$, and $\theta_{b}$ emitted in ${ }^{28} \mathrm{Si},{ }^{22} \mathrm{Ne},{ }^{16} \mathrm{O},{ }^{12} \mathrm{C}$, and ${ }^{4} \mathrm{He}$ interactions with $\mathrm{Em}$, together with their corresponding distributions obtained by the multisource thermal model, are given in Figures 5-7. It is evident from these figures that the values of $\theta_{s}, \theta_{g}$, and $\theta_{b}$ are nearly independent of the nature of incident projectiles. Figure 5 illustrated the angular distributions of shower particles. The curves are the distributions obtained by the model calculations. From Figure 5, it can be noticed that the peak increases with increase in projectile mass and the model is in agreement with experimental data in describing angular distributions of the shower particles.

The angular distributions of grey particles, $\theta_{g}$, are illustrated in Figure 6. For comparison, distributions obtained by the model calculations are also shown. The model agrees with experimental data in describing angular distributions for the grey particles. Also, it is notable that the angular distributions 


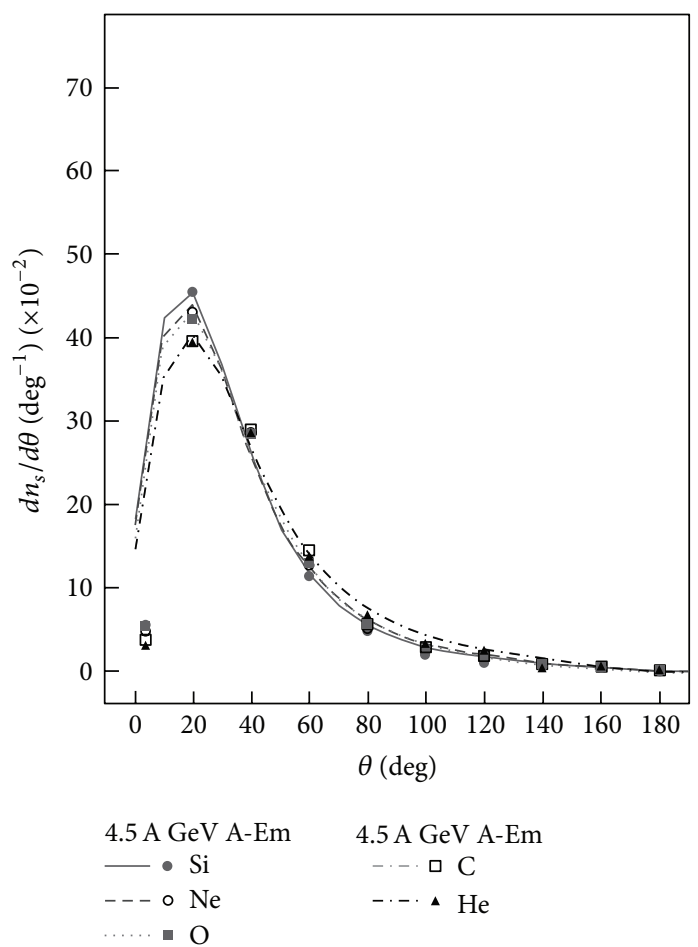

Figure 5: Angular distributions of shower particle produced in ${ }^{4} \mathrm{He}$ - (solid triangle), ${ }^{12} \mathrm{C}$ - (open square), ${ }^{16} \mathrm{O}$ - (solid square), ${ }^{22} \mathrm{Ne}-($ open circles), and ${ }^{28} \mathrm{Si}$ - (solid circles) Em collisions at (4.1-4.5) A GeV/c. The symbols represent the experimental data. Capone the curves are our calculated results by multisource thermal model.

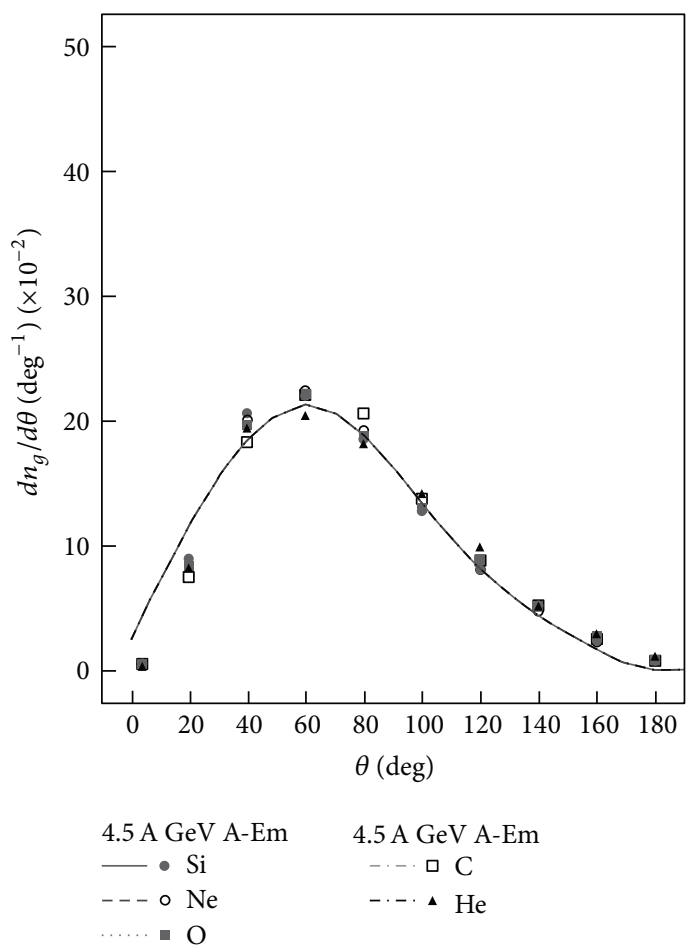

Figure 6: Angular distributions of grey particle produced in ${ }^{4} \mathrm{He}$ - (solid triangle), ${ }^{12} \mathrm{C}$ - (open square), ${ }^{16} \mathrm{O}$ - (solid square), ${ }^{22} \mathrm{Ne}-($ open circles), and ${ }^{28} \mathrm{Si}$ - (solid circles) Em collisions at (4.1-4.5) A GeV/c. The symbols represent the experimental data. Capone the curves are our calculated results by multisource thermal model. 
TABLE 5: Parameter values for multiplicity distributions for shower, grey, and black particles. The values of $\left\langle n_{i 1}\right\rangle,\left\langle n_{i 2}\right\rangle, m_{1}, m_{2}$, and $k_{1}, k_{2}$ for ${ }^{4} \mathrm{He}-,{ }^{12} \mathrm{C}-,{ }^{16} \mathrm{O}-$ - and ${ }^{28} \mathrm{Si}-\mathrm{AgBr}$ at $4.5 \mathrm{~A} \mathrm{GeV} / \mathrm{c}$ and ${ }^{22} \mathrm{Ne}-\mathrm{AgBr}$ at $4.1 \mathrm{~A} \mathrm{GeV} / \mathrm{c}$.

\begin{tabular}{|c|c|c|c|c|c|c|c|c|}
\hline Projectile & & $\left\langle n_{i 1}\right\rangle$ & $\left\langle n_{i 2}\right\rangle$ & $m_{1}$ & $m_{2}$ & $k_{1}$ & $k_{2}$ & $\chi^{2} / \mathrm{dof}$ \\
\hline \multirow{3}{*}{${ }^{4} \mathrm{He}$} & $n_{s}$ & 2.45 & 0.9 & 2 & 8 & 0.4 & 0.6 & 0.72 \\
\hline & $n_{g}$ & 1.23 & 2.3 & 5 & 6 & 0.5 & 0.5 & 0.878 \\
\hline & $n_{b}$ & 1.67 & - & 6 & - & 1 & 0 & 1.109 \\
\hline \multirow{3}{*}{${ }^{12} \mathrm{C}$} & $n_{s}$ & 2.6 & 2.4 & 3 & 8 & 0.42 & 0.58 & 0.702 \\
\hline & $n_{g}$ & 1.35 & 3.2 & 5 & 5 & 0.5 & 0.5 & 1.217 \\
\hline & $n_{b}$ & 1.67 & - & 6 & - & 1 & 0 & 0.744 \\
\hline \multirow{3}{*}{${ }^{16} \mathrm{O}$} & $n_{s}$ & 3.55 & 2.96 & 3 & 8 & 0.5 & 0.5 & 1.883 \\
\hline & $n_{g}$ & 1.28 & 2.5 & 6 & 8 & 0.56 & 0.44 & 1.983 \\
\hline & $n_{b}$ & 1.62 & - & 6 & - & 1 & 0 & 1.296 \\
\hline \multirow{3}{*}{${ }^{22} \mathrm{Ne}$} & $n_{s}$ & 6.3 & 5.25 & 2 & 5 & 0.5 & 0.5 & 1.102 \\
\hline & $n_{g}$ & 1.6 & 3.35 & 5 & 6 & 0.5 & 0.5 & 1.018 \\
\hline & $n_{b}$ & 1.58 & - & 6 & - & 1 & 0 & 1.083 \\
\hline \multirow{3}{*}{${ }^{28} \mathrm{Si}$} & $n_{s}$ & 5.8 & 6.3 & 2 & 5 & 0.5 & 0.5 & 1.146 \\
\hline & $n_{g}$ & 1.46 & 4 & 5 & 5 & 0.5 & 0.5 & 1.69 \\
\hline & $n_{b}$ & 2 & - & 5 & - & 1 & 0 & 1.127 \\
\hline
\end{tabular}

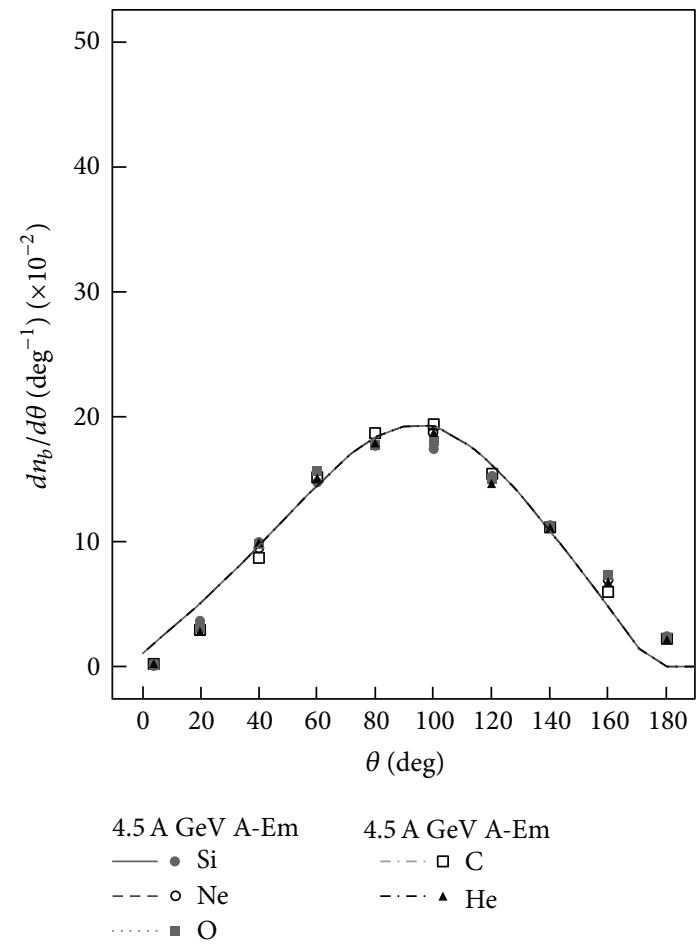

Figure 7: Angular distributions of black particle produced in ${ }^{4} \mathrm{He}$ - (solid triangle), ${ }^{12} \mathrm{C}$ - (open square), ${ }^{16} \mathrm{O}$ - (solid square), ${ }^{22} \mathrm{Ne}$ - (open circles), and ${ }^{28} \mathrm{Si}$ - (solid circles) Em collisions at (4.14.5) A GeV/c. The symbols represent the experimental data. Capone the curves are our calculated results by multisource thermal model.

of grey particles become wider than those of shower particles with displacement of the peak position to higher values of $\theta$.

Figure 7 illustrated the angular distributions of black particles, $\theta_{b}$. For comparison, distributions obtained by the
TABLE 6: The peak position of angular distributions for shower, grey, and black particles.

\begin{tabular}{llll}
\hline Projectile & $\theta_{s_{p}}$ & $\theta_{g_{p}}$ & $\theta_{b_{p}}$ \\
${ }^{4} \mathrm{He}$ & 20 & 60 & 90 \\
${ }^{12} \mathrm{C}$ & 20 & 60 & 90 \\
${ }^{16} \mathrm{O}$ & 20 & 60 & 90 \\
${ }^{22} \mathrm{Ne}$ & 20 & 60 & 90 \\
${ }^{28} \mathrm{Si}$ & 20 & 60 & 90 \\
\hline
\end{tabular}

multisource thermal model calculations are also shown. The model agrees with experimental data. It can be noticed that the angular distributions for black particles are nearly symmetrical around the peak position.

From Figure 5 to Figure 7, we observe that the angular distributions of shower, grey, and black particles produced are independent of projectile mass. The peak position shifts towards higher values of $\theta$ with increase in product mass, that is, $\theta_{s_{p}}<\theta_{g_{p}}<\theta_{b_{p}}$, which is clear in Table 6. The parameter values for angular distributions for shower, grey, and black particles $a_{x}, a_{y}$, and $a_{z}$ and $b_{x}, b_{y}$, and $b_{z}$ are given in Table 7 .

\section{Conclusion}

We conclude that multisource thermal model gives uniform description of the target fragmentation in interaction of ${ }^{4} \mathrm{He}$, ${ }^{12} \mathrm{C},{ }^{16} \mathrm{O},{ }^{22} \mathrm{Ne}$, and ${ }^{28} \mathrm{Si}$ with emulsion at $4.1-4.5 \mathrm{~A} \mathrm{GeV} / \mathrm{c}$. This model has succeeded in reproduction of the general characteristics of interactions of nuclei with emulsion such as average multiplicities, multiplicity distributions, and angular distributions of particles produced in N-N collisions. 
TABLE 7: Parameter values for angular distributions for shower, grey, and black particles. The values of $a_{x}, a_{y}$, and $a_{z}$ and $b_{x}, b_{y}$, and $b_{z}$ for ${ }^{4} \mathrm{He}-,{ }^{12} \mathrm{C}-,{ }^{16} \mathrm{O}$-, and ${ }^{28} \mathrm{Si}-\mathrm{Em}$ at $4.5 \mathrm{~A} \mathrm{GeV} / \mathrm{c}$ and ${ }^{22} \mathrm{Ne}-\mathrm{Em}$ at $4.1 \mathrm{~A} \mathrm{GeV} / \mathrm{c}$.

\begin{tabular}{|c|c|c|c|c|c|c|c|}
\hline Projectile & & $a_{x}$ & $a_{y}$ & $a_{z}$ & $b_{x}$ & $b_{y}$ & $b_{z}$ \\
\hline \multirow{3}{*}{${ }^{4} \mathrm{He}$} & $n_{s}$ & 1 & 1 & 1.3 & 0 & 0 & 1.7 \\
\hline & $n_{g}$ & 1.25 & 1 & 1 & 0 & 0 & 0.47 \\
\hline & $n_{b}$ & 1.2 & 1 & 1 & 0 & 0 & -0.15 \\
\hline \multirow{3}{*}{${ }^{12} \mathrm{C}$} & $n_{s}$ & 1 & 1 & 1.3 & 0 & 0 & 1.7 \\
\hline & $n_{g}$ & 1.25 & 1 & 1 & 0 & 0 & 0.47 \\
\hline & $n_{b}$ & 1.2 & 1 & 1 & 0 & 0 & -0.15 \\
\hline \multirow{3}{*}{${ }^{16} \mathrm{O}$} & $n_{s}$ & 1 & 1 & 1.3 & 0 & 0 & 1.85 \\
\hline & $n_{g}$ & 1.25 & 1 & 1 & 0 & 0 & 0.47 \\
\hline & $n_{b}$ & 1.2 & 1 & 1 & 0 & 0 & -0.15 \\
\hline \multirow{3}{*}{${ }^{22} \mathrm{Ne}$} & $n_{s}$ & 1 & 1 & 1.3 & 0 & 0 & 1.9 \\
\hline & $n_{g}$ & 1.25 & 1 & 1 & 0 & 0 & 0.47 \\
\hline & $n_{b}$ & 1.2 & 1 & 1 & 0 & 0 & -0.15 \\
\hline \multirow{3}{*}{${ }^{28} \mathrm{Si}$} & $n_{s}$ & 1 & 1 & 1.3 & 0 & 0 & 2 \\
\hline & $n_{g}$ & 1.25 & 1 & 1 & 0 & 0 & 0.47 \\
\hline & $n_{b}$ & 1.2 & 1 & 1 & 0 & 0 & -0.15 \\
\hline
\end{tabular}

\section{Conflict of Interests}

The authors declare that there is no conflict of interests regarding the publication of this paper.

\section{References}

[1] J. X. Sun, "Application of the multisource thermal model in pseudorapidity distributions of charged particles produced in $p \bar{p}$ or pp collisions over an energy range from 0.053 to $7 \mathrm{TeV}$," Indian Journal of Pure and Applied Physics, vol. 50, pp. 374-379, 2012.

[2] S. Sarfaraz Ali and H. Khushnood, "Some aspects of secondary charged particles produced in $4.5 \mathrm{~A} \mathrm{GeV} / \mathrm{c} 28 \mathrm{Si}$-nucleus interactions," Research Journal of Recent Sciences, vol. 1, pp. 232-237, 2012.

[3] A. Abd El-Daiem, M. Abdo Khalifa, and A. Abdel-hafiez, "Study of the central heavy-ion collision with emulsion at $4.5 \mathrm{~A} \mathrm{GeV} / \mathrm{c}$," Journal of Modern Physics, vol. 2, pp. 669-674, 2011.

[4] S. Fakhraddin, M. A. Rahim, and F.-H. Liu, "Anisotropic emission of final-state products in ${ }^{22} \mathrm{Ne}$-emulsion collisions at 4.1A GeV/c," Nuclear Physics A, vol. 858, no. 1, pp. 95-105, 2011.

[5] E. Q. Wang, F.-H. Liu, M. A. Rahim, S. Fakhraddin, and S. Jian-Xin, "Singly and doubly charged projectile fragments in nucleus-emulsion collisions at dubna energy in the framework of the multi-source model," Chinese Physics Letters, vol. 28, no. 8, Article ID 082501, 2011.

[6] M. A. Rahim and S. Fakhraddin, "Study of events in absence of target/projectile fragments in interactions of nuclei with emulsion at 4.5 and 4.1 A GeV/c," Nuclear Physics A, vol. 831, no. 1-2, pp. 39-48, 2009.

[7] M. S. Khan, P. P. Shukla, and H. Khushnood, "Nature of multiplicity correlations in ${ }^{12} \mathrm{C}$-nucleus reaction at $4.5 \mathrm{AGeV}$," in Proceedings of the DAE Symposium on Nuclear Physics, vol. 56, 2011

[8] S. Dhamji, M. M. Aggarwal, and V. S. Bhatia, "Slow particle production in the interactions of 200 a gev sulphur projectile with nuclear emulsion," Modern Physics Letters A, vol. 16, p. 1801, 2001.

[9] M. E. Solit and A. Abd El-Daiem, "Multiplicity distributions in $24 \mathrm{Mg}$-emulsion collisions at $4.5 \mathrm{~A} \mathrm{GeV/c,"} \mathrm{International} \mathrm{Journal}$ of Physical Sciences, vol. 2, no. 12, pp. 324-330, 2007.

[10] S. Fakhraddin, "Study of pseudorapidity distributions of shower particles emitted in interactions of nuclei with emulsion at 4.14.5 A GeV/c," Turkish Journal of Physics, vol. 29, no. 2, pp. 79-84, 2005.

[11] F. H. Liu, "Target black fragment flows in magnesium-emulsion collisions at 4.5A GeV/c," Radiation Measurements, vol. 43, pp. S234-S238, 2008.

[12] A. A. El-Daiem, "Target fragmentation at $4.5 \mathrm{a} \mathrm{GeV} / \mathrm{c}$ in ${ }^{24} \mathrm{Mg}$ and ${ }^{28} \mathrm{Si}$ with emulsion interaction," American Journal of Applied Sciences, vol. 6, no. 4, pp. 608-615, 2009.

[13] A. Abd El-Daiem, "Characteristics of compound multiplicity in ${ }^{24} \mathrm{Mg}$ with emulsion at $4.5 \mathrm{~A} \mathrm{GeV} / \mathrm{c}$," Physics International, vol. 1, no. 1, pp. 31-37, 2010.

[14] N. N. Abd-Alaah and M. Mohery, "Features of the compound multiplicity of the interactions of ${ }^{24} \mathrm{Mg}$ and ${ }^{28} \mathrm{Si}$ ions with emulsion nuclei at 4.5A GeV/c," Turkish Journal of Physics, vol. 25, pp. 109-119, 2001.

[15] B. K. Singh and S. K. Tuli, "Central collisions of ${ }^{28}$ Si with $\mathrm{Ag}(\mathrm{Br})$ nuclei," Nuclear Physics A, vol. 602, no. 3-4, pp. 487-501, 1996.

[16] F. H. Liu, "Target fragmentation in oxygen-emulsion collisions at dubna and SPS energies," Chinese Journal of Physics, vol. 40, no. 2, 2002.

[17] F. H. Liu, "Emission of relativistic light fragments in nucleusemulsion collisions at high energy," Chinese Journal of Physics, vol. 38 , no. 6,2000 .

[18] H. L. Li, D. H. Zhang, and J. S. Li, "Multiplicities of forwardbackward particles in ${ }^{16} \mathrm{O}$-emulsion interactions at $4.5 \mathrm{AGeV} / \mathrm{c}$," Chinese Physics C, vol. 33, no. 7, pp. 521-527, 2009.

[19] A. Abdelsalam and B. M. Badawy, "About target fragmentation in nuclear emulsion at relativistic energy," Journal of Nuclear and Radiation Physics, vol. 3, no. 2, pp. 109-121, 2008. 
[20] F. H. Liu, N. N. Abd Allah, B. K. Singh, and T. Ahmad, "angular distributions of target fragments in ${ }^{84} \mathrm{kr}$-em and ${ }^{16} \mathrm{O}-$ em collisions at high energies," Acta Physica Polonica B, vol. 37, no. 9, 2006.

[21] B. Bhattacharjee, A. Mukhopadhyay, V. Singh, S.K. Tuli, and S. Sengupta, "On the emission of fast and slow target fragments from ${ }^{84} \mathrm{Kr}-\mathrm{AgBr}$ interactions at $0.95 \mathrm{GeV} / \mathrm{A}$," Radiation Measurements, vol. 36, pp. 291-294, 2003.

[22] M. A. Jilany, "Nuclear fragmentation in interactions of 3.7A $\mathrm{GeV}^{24} \mathrm{Mg}$ projectiles with emulsion targets," Physical Review $\mathrm{C}$, vol. 70, no. 1, Article ID 014901, 2004.

[23] A. Abdelsalam, M. S. El-Nagdy, N. Rashed, and B. M. Badawy, "Signatures of target fragmentation of nuclear emulsion by light nuclei," Chinese Journal of Physics, vol. 45, no. 3, 2007.

[24] B. M. Badawy, "Central collisions induced by light target nuclei," Journal of Nuclear and Radiation Physics, vol. 3, no. 1, pp. 31-51, 2008.

[25] Z. Dong-Hai, L. Fang, H. Chun-Le et al., "Features of the total disintegration events of heavy emulsion targets caused by $4.5 \mathrm{~A}$ GeV/c 16O," Chinese Physics, vol. 1, no. 11, pp. 2564-2570, 2006.

[26] A. Abdelsalam, M. S. El-Nagdy, N. Rashed, and B. M. Badawy E -El Falaky, "Further study on grey particles production in $3 \mathrm{He}$ and $4 \mathrm{He}$ interactions with emulsion at $4.5 \mathrm{~A} \mathrm{GeV} / \mathrm{c}$," Journal of Nuclear and Radiation Physics, vol. 2, no. 1, pp. 49-60, 2007.

[27] A. Al-Haydari and M. T. Hussein, "The multiplicity of particle production from hadronhadron and nucleus-nucleus interaction," Natural Science, vol. 2, no. 1, pp. 388-397, 2010.

[28] L. G. Moretto, L. Phair, and G. J. Wozniak, "Resilient reducibility in nuclear multifragmentation," Physical Review C, vol. 60, no. 3, Article ID 031601, 1999.

[29] C. Hartnack, L. Zhuxia, L. Neise et al., "Quantum molecular dynamics a microscopic model from UNILAC to CERN energies," Nuclear Physics A, vol. 495, pp. 303-319, 1989.

[30] R. G. Nazmitdinov, E. I. Shahaliev, M. K. Suleymanov, and S. Tomsovic, "Analysis of nucleus-nucleus collisions at high energies and random matrix theory," Physical Review C, vol. 79, Article ID 054905, 2009.

[31] F. H. Liu, "Unified description of multiplicity distributions of final-state particles produced in collisions at high energies," Nuclear Physics A, vol. 810, pp. 159-172, 2008.

[32] F. H. Liu, "Longitudinal and transverse flows of protons in 28AGeVAu-Au collisions," European Physical Letters, vol. 63, p. 193, 2003.

[33] F. H. Liu, N. N. Abd-Allah, D.-H. Zhang, and M.-Y. Duan, "angular distributions of target black fragments in nucleusnucleus collisions at high energy," International Journal of Modern Physics E, vol. 12, p. 713, 2003.

[34] F.-H. Liu, N. N. A. Allah, and B. K. Singh, "Dependence of black fragment azimuthal and projected angular distributions on polar angle in silicon-emulsion collisions at $4.5 \mathrm{~A} \mathrm{GeV} / \mathrm{c}$," Physical Review C, vol. 69, no. 5, Article ID 057601, 2004.

[35] F.-H. Liu, Q.-W. Lu, B.-C. Li, and R. Bekmirzaev, "A description of the multiplicity distributions of nuclear fragments in hA and AA collisions at intermediate and high energies," Chinese Journal of Physics, vol. 49, pp. 601-620, 2011.

[36] F. H. Liu, J. S. Li, and M. Y. Duan, "Light fragment emission in ${ }^{86} \mathrm{Kr}^{-}{ }^{124}$ Sn collisions at $25 \mathrm{MeV} /$ nucleon," Physical Review $\mathrm{C}$, vol. 75, Article ID 054613, 5 pages, 2007.

[37] Z. Sun, "Unified description of angular distributions of target particles in light and heavy ion induced interactions in nuclear emulsions at high energies," Chinese Physics C, vol. 33, pp. 256$262,2009$.
[38] M. A. Rahim, S. Fakhraddin, and H. Asharabi, "Systematic study of projectile fragments in nucleus-nucleus collisions at 4.1-4.5 A GeV/c and multi-source thermal model ", European Physical Journal A, vol. 48, article 115, 2012.

[39] S. Fakhraddin and M. A. Rahim, "Multiplicity distributions of projectile fragments in interactions of nuclei with emulsion at 4.1-4.5 A GeV/c," Physica Scripta, vol. 78, Article ID 015101, 2008.

[40] S. Fakhraddin, Study of interactions of nuclei with emulsion at high energies [Ph.D. thesis], Cairo University, Cairo, Egypt, 2000. 

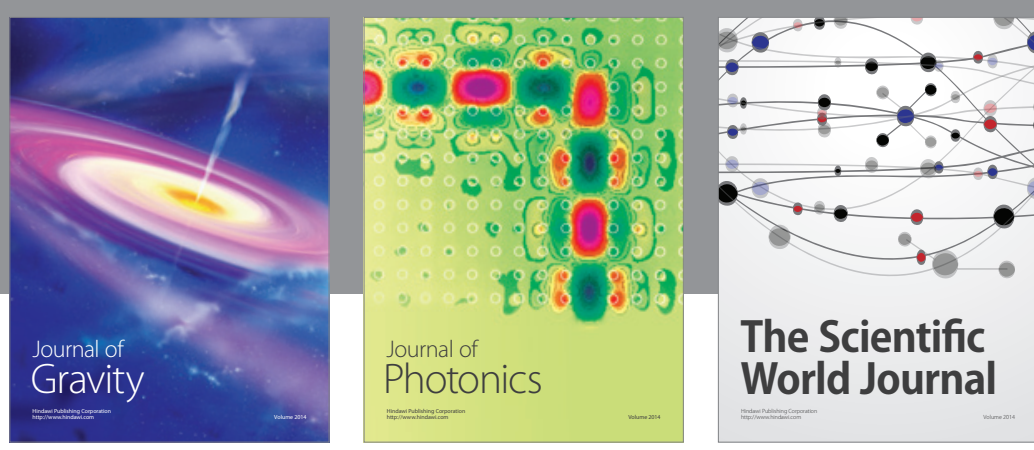

The Scientific World Journal
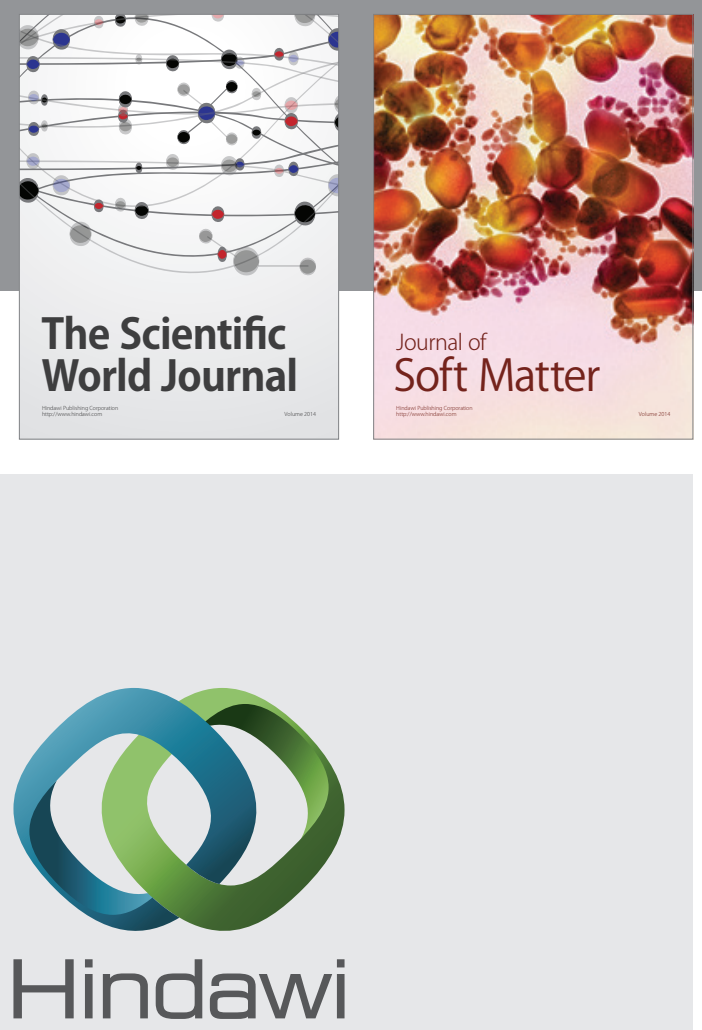

Submit your manuscripts at

http://www.hindawi.com

nternational Journal of

Statistical Mechanics
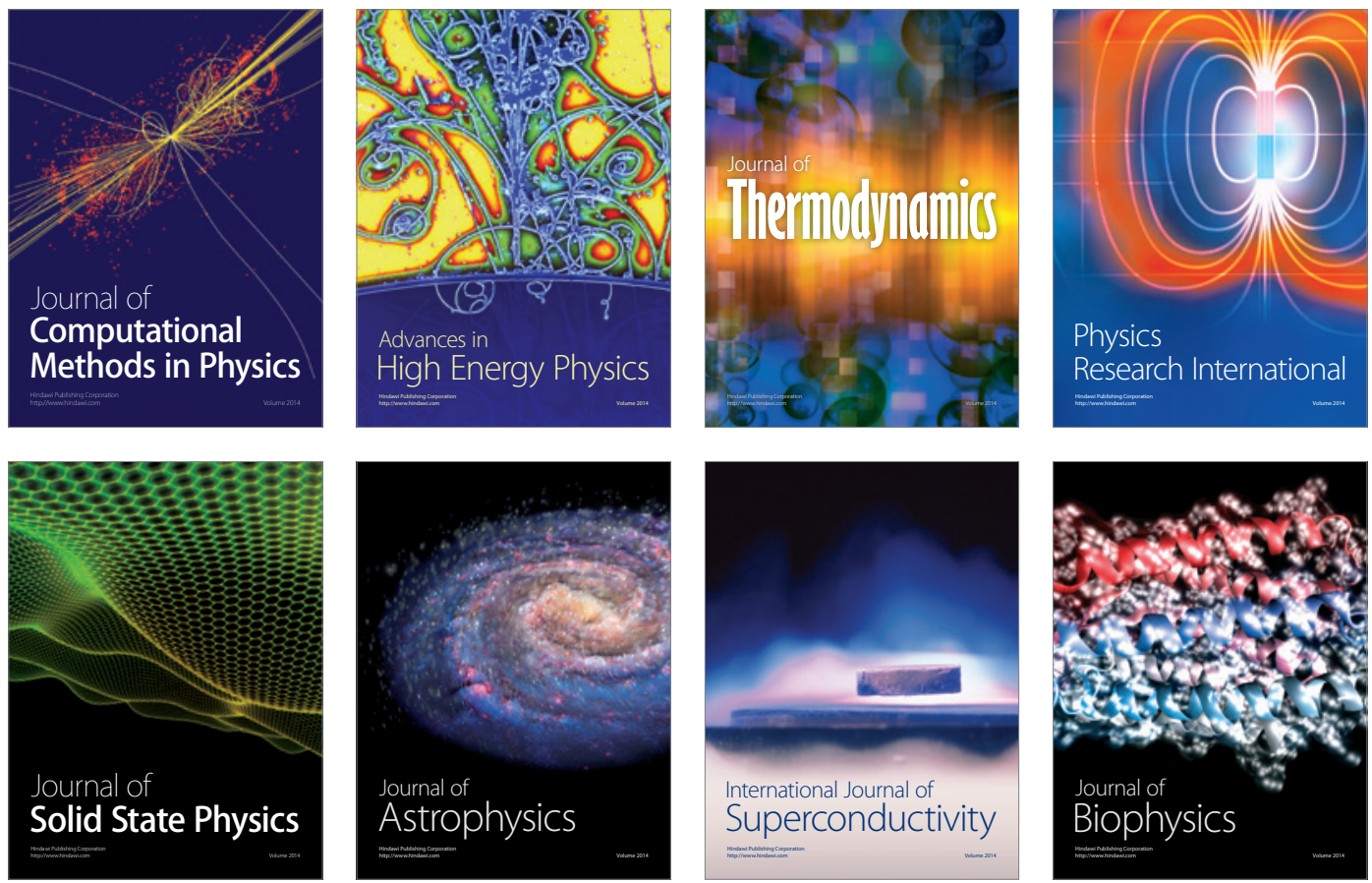
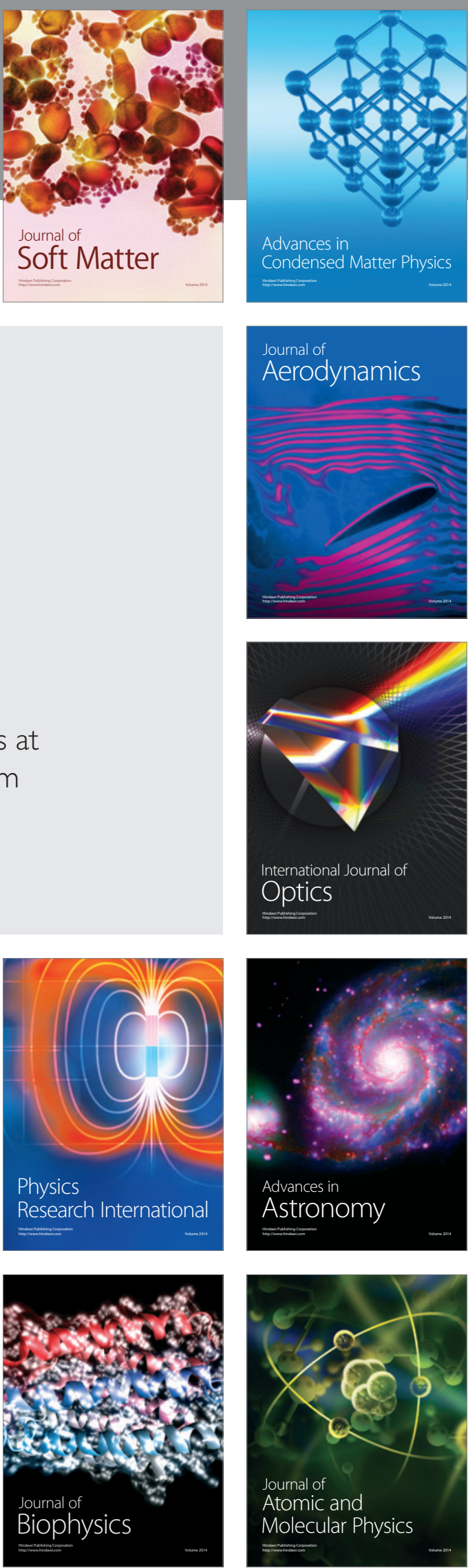